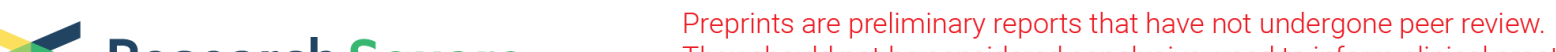 $\begin{array}{ll}\text { Research Square } & \text { They should not be considered conclusive, used to inform clinical practice, } \\ \text { or referenced by the media as validated information. }\end{array}$
}

\section{Standardized Stimulation Protocols Are Needed for Comparable Analysis of Pain-related Evoked Potentials (PREP)}

\section{Katharina Brech}

Ruhr-University Bochum, BG University Hospital Bergmannsheil gGmbH

\section{Elena K. Enax-Krumova}

Ruhr-University Bochum, BG University Hospital Bergmannsheil gGmbH

Lynn Eitner

Ruhr-University Bochum, University Children's Hospital

Jan Vollert

Imperial College London

\section{Christoph Maier}

Ruhr-University Bochum, University Children's Hospital

\section{Oliver Höffken}

Ruhr-University Bochum, BG University Hospital Bergmannsheil gGmbH

\section{Martin Tegenthoff}

Ruhr-University Bochum, BG University Hospital Bergmannsheil gGmbH Özüm Simal Özgül ( $\square$ oezuem.oezguel@rub.de)

Ruhr-University Bochum, BG University Hospital Bergmannsheil gGmbH

\section{Research Article}

Keywords: Pain-related evoked potentials (PREP), N1P1-amplitudes, N1-latencies, electrodes

Posted Date: November 30th, 2021

DOI: https://doi.org/10.21203/rs.3.rs-1079833/v1

License: (1) (1) This work is licensed under a Creative Commons Attribution 4.0 International License. Read Full License 


\section{Abstract}

Objective: Pain-related evoked potentials (PREP) are increasingly used to investigate nociception and small-fibre function. Due to lack of a standard stimulation protocol, it is unclear whether results from studies using different protocols are comparable. Aim of the study was to assess the influence of different stimulation parameters on N1P1-amplitudes, N1-latencies and PREP-induced pain intensity.

Methods: In a cross-over design we examined 31 healthy volunteers using four different stimulation protocols (number of stimulation electrodes 1 vs. 3, stimulus durations $200 \mu \mathrm{s}$ vs. $500 \mu \mathrm{s}$ ) in a randomized order. Statistics: paired t-test, ANOVA, correlation analyses.

Results: Longer stimulus duration induced higher N1P1-amplitudes $(p<0.05)$ and higher pain intensity $(p<0.001)$. Stimulation with 3 electrodes lead to a lower pain intensity $(p<0.01)$, whereas the N1P1amplitude and stimulus intensity at twofold of individual pain remained unaffected by the number of electrodes. Also, there was no relation between stimulus intensities and N1P1-amplitudes (one electrode: $r=0.079 ; p=0.646$, three electrodes: $r=-0.10, p=0.70$ ) was observed. N1-latencies remained comparable between the four protocols.

Conclusions and Significance: The use of different stimulation protocols for PREP is limited by relevant differences in the N1P1-amplitudes and evoked pain intensities. Standard consented stimulation protocols are needed to allow data comparison between different labs and studies.

\section{Introduction}

Pain-related evoked potentials (PREP) allow the evaluation of electrophysiological stimulus transmission

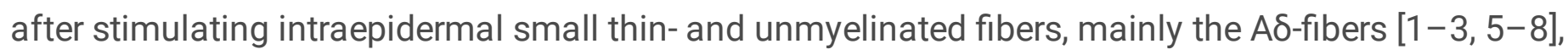
though the discussion about their specificity is still ongoing [1, 9]. A specially designed concentric electrode (K2-electrode) [2, 4] with a small anode-cathode distance resulting in a high current density despite low current intensities is being used for PREP recordings. Thus, only the nerve fibre endings of nociceptive fibres within the superficial skin layers are being depolarised, whilst avoiding activation of $A \beta-$ fibers in deeper layers $[2,10]$. PREPs have been shown to reflect somatosensory evoked cortical and insular summation potentials processing nociceptive information $[6,9,11,12]$.

Numerous studies using PREP investigated a wide range of diseases, for example small fibre neuropathies [13, 14], HIV-related neuropathy [3, 15], painful diabetic neuropathy [16], trigeminal neuralgia [17], M. Fabry [18], fibromyalgia [19, 20], mixed-fibre neuropathies [14, 21], sensory neuropathy in patients with cryoglobulin negative hepatitis- $C$ [5], medication overuse induced headache [6], peripheral nerve injury [22], and neuropathic pain [23]. Prolonged latencies and diminished amplitudes have been reported in neuropathic conditions $[16,20]$ compared to healthy controls [10]. High test-retest reliability regarding N1-latencies and N1P1-amplitudes has been confirmed [24], further strengthening its applicability as diagnostic tool. A significant correlation between the reduction of PREP-amplitudes and pain ratings has been reported in several studies $[2-4,10,11]$. Katsarava et al. reported that both PREP-amplitudes and 
pain intensity increase due to spatial (i.e. stimulation with several electrodes) and temporal (i.e. multiple pulse stimulation) summation concluding that PREPs are closely associated with pain perception [2].

However, experimental protocols and electrophysiological settings, especially the number of electrodes and the duration or number of monopolar square waves, vary between different studies, therefore compromising comparability $[2-6,10,15,18,20-24,33]$. For increasing the sensitivity to detect incomplete nerve lesions [22] by spatial summation our working group uses three parallel connected stimulation electrodes, applying triple-train of monopolar square waves (each lasting for $200 \mu \mathrm{s}$, interwave-interval $5 \mathrm{~ms}$ ) at the twofold of the individual pain threshold with interstimulus intervals of 1218 s $[24,25]$. This protocol allows the stimulation of a wider area to detect PREP changes caused by such incomplete nerve lesions. In contrast, other protocols used one [14,19-21] or two [3] electrodes and a triple-train stimulus with a square wave duration of $500 \mu \mathrm{s}$, or one [10] or two $[2,5,16]$ electrodes and a doble-train stimulus with a square wave duration of $500 \mu$ s. The impact on PREP parameters merely caused by changes in stimulation settings have not yet been explored in detail. Therefore, this study aimed to investigate four different protocols in healthy volunteers varying in stimulus duration (i.e. $200 \mu \mathrm{s}$ vs. $500 \mu \mathrm{s}$ ) and numbers of stimulation electrodes (1 vs. 3 electrodes) to analyse their influence on the commonly reported PREP parameters (N1P1-amplitudes and N1-latencies as well as evoked pain intensity).

\section{Material And Methods 2.1 Subjects}

The study was approved by the local ethics committee of the Faculty of Medicine, Ruhr University Bochum, (Reg. Nr.16-5664, 14.10.2017) and was performed in accordance with the Declaration of Helsinki. Healthy volunteers older than 18 years were recruited between March and June 2018 in the Department of Neurology, University Hospital Bergmannsheil gGmbH Bochum in Germany. Written informed consent was obtained from all participants before study inclusion.

Inclusion criteria were according to the published recommendations for healthy volunteers in studies on the somatosensory function [26]. Subjects were excluded if any of the following criteria were fulfilled: any pain experienced within the last four weeks with an intensity higher than 2/10 on the NRS (NRS $0-10,0=$ no pain, 10 = worst imaginable pain), history of radiculopathies, neuropathies or nerve injuries, topical treatment with local anaesthetic within the last six weeks, any anticonvulsive medication, any psychiatric diseases any neurological comorbidities such as multiple sclerosis, Parkinson's disease, migraine, dementia, major vascular diseases e.g. peripheral arterial occlusive disease, diabetes mellitus or any substance abuse such as alcohol, nicotine or drugs, insufficient German language skills. Additionally, a screening for neuropathic symptoms [28] as well as depressive symptoms [29] was conducted to detect subclinical symptoms. 
Age, sex, height and weight were recorded. The participants handedness was assessed by the Edinburgh Handedness Inventory [27].

Subjects were randomized into four groups (A-D), which underwent the experimental protocol (stimulus duration either $200 \mu$ s or $500 \mu$ s; either 1 or 3 stimulation electrodes) in different order as illustrated below in Fig. 1.

\subsection{Experimental setup}

Participants were seated in a relaxed position, with opened eyes and were instructed to avoid movements to prevent artefacts. The recordings were performed in a faraday cage, in order to minimise disruptive elements.

PREPs were electrically elicited at the foot dorsum, according to the participants' handedness, using four custom-built concentric electrodes (Inomed Medizintechnik GmbH, Lübeck, Germany) [1, 27], three of them being activated simultaneously. These were arranged triangularly at the medial foot dorsum within a distance of 2-2,5 cm from each other. A single electrode, which could be activated separately, was placed in the middle. The design of the electrodes assembled a central metal cathode (D: $0.5 \mathrm{~mm}$ ), an isolation insert (D: $10 \mathrm{~mm}$ ) and external anode (D: $6 \mathrm{~mm}$ ) [27], in total reaching a diameter of approximately $20 \mathrm{~mm}$ [23]. The single and the three parallel electrodes where connected to two separate constant current stimulators (Digitimer DS7A, Welwyn Garden City, UK) [15, 18].

For PREP recording a needle electrode (acupuncture needle, Hwato Master Touch $0.20 \times 15 \mathrm{~mm}$, for single use) was placed subcutaneously on $\mathrm{Cz}$ (vertex), which was referenced to linked ear lobes (A1, A2) according to the international 10-20 system. The ground electrode was placed on the forehead.

\subsection{Stimulation paradigm}

Before starting PREP recording, several electrical stimuli were delivered to familiarise the participant and adjust current intensities. Detection (DT) and pain thresholds (PT) were determined by a staircase procedure of $0.2 \mathrm{~mA}$ steps increases and decreases of stimulus energies, until subjects reported a sensation (DT) or a pinprick-like stinging (PT) $[2,24,25]$. They were ascertained separately for one and three electrodes, using stimulus duration of $200 \mu \mathrm{s}$. No threshold reassessment for stimulation with a stimulus duration of $500 \mu$ s was conducted.

Blocks of 20 stimuli (Fig. 2) with the same combination of stimulation settings were elicited at the foot dorsum of the side of the participants' handedness and were recorded separately.

Recording one block with 20 stimuli lasted for 4.5 minutes, separated by a 2-minute interval. Each PREP stimulus consisted of a triple-train ( 3 successive monopolar square waves: duration either $200 \mu \mathrm{s}$ or 500 $\mu s$; inter-wave interval within the triple-train $5 \mathrm{~ms}$; interstimulus interval: 12 - $18 \mathrm{~s}$ ). Pain intensity was rated after every forth stimulus, using a numerical rating scale $(0-100)$, and the five values later averaged. 


\subsection{Data storage and analysis}

Storage for offline analysis was achieved by a 32-channel-amplifier (Brain Amp, Brain Products, Germany; Bandwidth: $1 \mathrm{~Hz}-1 \mathrm{kHz}$; digitization sampling rate: $2.5 \mathrm{kHz}$ ). Offline analysis was carried out in sweeps between 200 ms before and 800 ms after stimulus onset, employing Vision Recorder Version 1.03. According to previous studies $[2,3,15-18,20,30]$ the first potential of every block was rejected in order to diminish any bias caused by a startling response. The remaining nineteen potentials were averaged, N1and P1-peaks of each averaged potential were determined manually as shown in Fig. 3. N1P1-amplitudes as well as N1-latencies were assessed for statistical analysis

\subsection{Statistical Analysis}

Mean values and standard deviations were calculated for N1-latency and N1P1-amplitudes as well as pain intensity ratings. Before using parametric tests, normal data distribution was confirmed by the Kolomogorov-Smirov test and homogeneity of variances was confirmed by a Levene test. In case of significance of these tests, we planned to use non-parametric analogous tests, following a similar procedure as described below for parametric tests. Further analysis was performed using SPSS (Version 20, IBM, Chicago, IL, USA). In order to analyse associations between N1-latency or N1P1-amplitude and demographical factor as well as stimulation intensities, two-paired Pearson's Correlations were used. The influence of randomization order, number of electrodes, and stimulus duration on N1-latency, N1P1amplitude and pain ratings was investigated with univariate ANOVA. For all calculations, significance was assumed at $a=0.05$.

\section{Results}

\subsection{Subjects demographic characteristics}

We included 34 healthy subjects ( 21 female, 13 male; mean age $26.7 \pm 9.5$ years range 18 to 53 years). In 30 subjects $(88 \%)$ testing took place at the right foot, in 4 subjects $(12 \%)$ at the left. Two subjects had to be excluded because of the absence of reproducible potentials. In addition, one participant was excluded due to fainting because of his needle phobia. Thus, 31 subjects ( 19 female, 12 male; mean age $26.5 \pm 9.4$ years, range 18 to 53 years) were included in the analysis.

\subsection{Detection and Pain thresholds}

ANOVA showed no significant effect of the number of electrodes on stimulation intensities when determining the detection and pain thresholds $(F=0.054, p=0.818)$.

When the detection thresholds were determined, there was no difference between the stimulus intensity using one electrode $(0.9 \pm 0.3 \mathrm{~mA}, 0.5-1.6 \mathrm{~mA})$ or three electrodes $(0.9 \pm 0.3 \mathrm{~mA}, 0.4-1.6 \mathrm{~mA})$. Pain thresholds were elicited at mean intensities of $2.8 \pm 0.9 \mathrm{~mA}(1.2-4.4 \mathrm{~mA})$ using one electrode and $2.8 \pm$ $1.1 \mathrm{~mA}(1.2-5.6 \mathrm{~mA})$ using three electrodes. Hence, stimulation currents needed for testing at the twofold of pain intensities were $5.5 \pm 2.0 \mathrm{~mA}$ and $5.5 \pm 2.1 \mathrm{~mA}$, correspondingly. 


\subsection{PREP-latencies, -amplitudes and pain ratings}

Table 1 summarizes the relevant data for each stimulation combination.

Table 1

Mean-values and standard deviation of N1-latencies [ms], N1P1-amplitudes [ $\mu \mathrm{V}]$ and evoked pain [NRS] for each stimulus combination.

\begin{tabular}{|c|c|c|c|c|}
\hline & $1 \mathrm{E} / 200 \mu \mathrm{s}$ & $1 \mathrm{E} / 500 \mu \mathrm{s}$ & $3 E / 200 \mu s$ & $3 \mathrm{E} / 500 \mu \mathrm{s}$ \\
\hline N1-latency [ms] & $159 \pm 37$ & $154.8 \pm 26.7$ & $149.1 \pm 35.6$ & $154.6 \pm 32.7$ \\
\hline N1P1-amplitude $[\mu \mathrm{V}]$ & $30.5 \pm 12.3$ & $36.2 \pm 18$ & $29.2 \pm 10.9$ & $33.1 \pm 13.1$ \\
\hline Evoked pain [NRS] & $42.2 \pm 22.5$ & $60.2 \pm 21.0$ & $32.8 \pm 17.8$ & $53.0 \pm 19.6$ \\
\hline
\end{tabular}

ANOVA of N1P1-amplitude ( $F=1.453, p=0.232)$, N1-latency $(F=0.246, p=0.861)$, or evoked pain intensity $(F=1.106, p=0.351)$, as variable factor and "randomization order" as independent variable could not sustain a statistically significant sequence effect comparing the data of the four randomised groups A-D. Thus all data were analysed independently from the sequence.

A significant effect of the stimulus duration was found for N1P1-amplitudes, and the evoked pain intensity (Table 2). A stimulus duration of $500 \mu$ s led to higher amplitudes and higher evoked pain ratings than the stimulus duration of $200 \mu$ s (Table 2). There was no interaction between stimulus duration and N1-latencies (Table 2).

Table 2

ANOVA for PREP parameters with the factors stimulus duration and number

of electrodes. Bold: $p$-value below the level of significance

\begin{tabular}{|lllll|}
\hline & \multicolumn{2}{l}{ stimulus duration } & \multicolumn{2}{l|}{ number of electrodes } \\
\cline { 2 - 5 } & F-value & significance & F-value & significance \\
\hline N1P1-amplitude & $F=4.063$ & $\mathbf{p}=\mathbf{0 . 0 4 6}$ & $F=1.865$ & $p=0.175$ \\
N1-latency & $F=0.629$ & $p=0.429$ & $F=0.390$ & $p=0.533$ \\
\hline Evoked pain & $F=29.22$ & $\mathbf{p}<\mathbf{0 . 0 0 1}$ & $F=7.289$ & $\mathbf{p}=\mathbf{0 . 0 0 8}$ \\
\hline
\end{tabular}

The variation of the number of electrodes did not show a significant effect on the N1P1-amplitude, but evoked pain ratings were significantly different. Stimulating with 3 electrodes induced lower evoked pain intensities than using only one electrode (Table 1). The number of electrodes had neither a significant effect on the N1-latencies nor on the stimulus intensities $(F=0.054, p=0.818)$. 
The stimulus intensities showed a significant correlation neither with the N1-latency $(r=-0.138, p=0.474$ for 1 electrode; $r=0.217, p=0.244$ for 3 electrodes) nor with the N1P1-amplitude $(r=0.079, p=0.671$ for 1 electrode; $r=-0.10, p=0.70$ for 3 electrodes), or with the evoked pain intensity $(r=0.332, p=0.862$ for 1 electrode; $r=-0.128, p=0.49$ for 3 electrodes).

\section{Discussion}

The current study compared different stimulus durations and number of electrodes to determine the influence of these factors on N1P1-amplitudes, N1-latencies and evoked pain intensity. To summarize, longer stimulus duration induced significantly higher N1P1-amplitudes and higher pain intensity than short stimulus duration. Stimulation with 3 electrodes lead to a significantly lower pain intensity than stimulation with one electrode, whereas the N1P1-amplitude remained unaffected. Neither variation of stimulus duration nor of number of electrodes had a significant effect on N1-latencies.

Currently, there is no standard stimulation protocol for PREP. Previous studies differ in stimulation protocols using different numbers of electrodes, different stimulus durations or a different number of square waves $[2,3,4,5,6,10,15,18,20,21,33]$. Thus, comparability between studies may be compromised and the effects of different stimulation parameters on PREP readouts are unknown. Thereby, our focus laid on N1-latency and N1P1-amplitude as they have been shown to have a diagnostic value, e.g. N1-latencies were prolonged and N1P1-amplitudes reduced in small fibre neuropathies [3, 16, 18]). N1P1-amplitudes have been proposed as a more specific nociceptor marker than latencies due to their similarity to amplitudes, morphology and topographic localization with respect to well established and validated LEPs [18]. Due to their ability to distinguish between healthy controls and patients, they serve as primary targets for PREP studies [5, 10, 13, 16, 18, 20, 29, 33].

Effects of different stimulus duration

In previous studies PREP were performed with a stimulus duration of a square wave of $500 \mu s$ using a double- $[10,15,16]$ or triple-train $[3,5,14,19-21]$ stimulation. In our previous studies we stimulated with a triple-train stimulus with a duration of $200 \mu s$ [22, 23, 24], aiming to minimize PREP-induced pain intensity and increase tolerability. Comparing a monopolar square wave duration of $200 \mu s$ vs. $500 \mu s$, we have now found a significant impact of stimulus duration on both N1P1-amplitudes and PREP-evoked pain intensity but not on N1-latency. The N1P1-amplitudes during stimulation at twice the pain threshold at $500 \mu$ s stimulation time were higher than those at $200 \mu s$ stimulation. This might be interpreted as a result of temporal summation. Previous studies varied not the stimulation duration, but the number of rectangular stimuli reported similar findings [1,30]. Both variations lead ultimately to change in the general current flow. Comparing the results of N1P1-amplitudes (39 $\pm 31 \mu \mathrm{V})$ of controls after footstimulation in the study of Hansen et al. [4], who used one electrode and a triple-train stimulation with the N1P1-amplitudes $(18 \pm 8.3 \mu \mathrm{V})$ to the study of Oh et al. [10], who stimulated with one electrode and a double-train, we can observe non-negligible differences in amplitude levels. Even in other works when two electrodes and a double-train was used (25.3 $\pm 9.1 \mu \mathrm{V}[16] ; 24.3 \pm 8,9 \mu \mathrm{V}[15] ; 23.7 \pm 7.3 \mu \mathrm{V}$ [5]) N1P1- 
amplitudes were lower, indicating that the height of the amplitudes depends on temporal summation. Thus, comparison of the results of PREP amplitudes between different protocols is limited.

\section{Effects of different number of electrodes}

The number of electrodes differed between previous studies, using one $[10,14,19-21]$ or two $[3,5,15,16]$ electrodes. To cover a larger area of skin to detect peripheral nerve damage in a better way, in our previous studies we have used a stimulation protocol with 3 electrodes $[22,23,24]$.

When eliciting PREP with the twofold of the pain threshold, the PREP amplitudes remained unaffected by the number of electrodes (one vs. three). When using 3 electrodes in parallel connection more fibers are stimulated but with a lower current intensity. This seems to elicit potentials with constant amplitudes. The lower current intensity ultimately causes a lower pain sensation. The expected effect of spatial summation [2, 31, 32], thus implying that the three electrode approach would yield higher amplitudes as various afferents converge imposing synchronisation effects [32], could not be confirmed by our data. We assume that in those studies, where there were hints for a spatial summation, the electrode arrangement was probably not connected in parallel but in series, which unfortunately has not been reported in the previous studies.

Furthermore, there was no significant difference in individual pain thresholds and therefore also in stimulation intensities when using one or three electrodes, despite the expected different current flows. We attribute this to the fact that the stimulation intensity for determining the pain threshold is in a low range $(2.8 \pm 0.9 \mathrm{~mA}$ for one and $2.8 \pm 1.1 \mathrm{~mA}$ for three electrodes $)$.

\section{Effects of stimulus intensity}

As we could show in our earlier works N1P1-amplitudes and N1-latency as well as the evoked pain intensity remained unaffected by stimulus intensities. Compared to other working groups stimulating the feet (e.g. 1.7 (0.6-2.4) mA [18], $1.8 \pm 0.4 \mathrm{~mA}$ [14]), we obtained a higher stimulation intensity $(5.5 \pm 2.0 \mathrm{~mA}$ for 1 electrode, and $5.5 \pm 2.1 \mathrm{~mA}$ for 3 electrodes) required to perform PREP after determining the individual pain thresholds. The difference is explainable by the lower rectangular wave duration of 200 $\mu s$. Theoretically, a higher current flow by using higher stimulation intensities can increase the current depth to such an extent that faster fibers are also excited [34]. However, the special design of the concentric K2-electrode produces a superficial current flow with a higher current density even at higher stimulation intensities. Unchanged N1P1-ampitudes and N1-latencies even when stimulating with higher intensities speaks for the fact that the same fiber systems - primarily a-delta-fibers - are obtained. Our N1-latencies (min. $149.2 \pm 35.6 \mathrm{~ms}$, max. $159.0 \pm 37.0$ ) are comparable to those in other studies (162 \pm $31 \mathrm{~ms}$ [4] to $180.3 \pm 15.2 \mathrm{~ms}$ [5]).

Diagnostic implications

However, the fact that a change in pain intensity can occur independently of the magnitudes of the amplitude questions the previous hypothesis that PREP can be a diagnostic tool reflecting the evoked 
pain intensity [2]. However, PREP is suitable for investigations of small fiber function in peripheral nerve injuries or polyneuropathies since the use of several electrodes have no effect on the magnitude of the amplitudes. Magnitude of PREP amplitudes would rather reflect the intraepidermal small fibre density or function, independently from the evoked pain intensity. Thus, stimulation with 3 electrodes covering a larger skin area and recruiting a higher number of small fibers would increase the sensitivity to detect a possible small fiber affection. Based on our data, we would recommend to use a stimulation duration of $500 \mu$ s as square wave duration using a triple-train stimulus to avoid high stimulation intensities.

Finally, stimulation standards must be defined to allow comparison between studies using PREP as a marker for small fiber function. Further studies investigating the application of more concentric electrodes to elicit PREP in patients with suspected neuropathy are needed. In addition, studies comparing PREP in patients with painful vs. non-painful small fiber neuropathies are rare.

\section{Conclusions}

To summarise, our study confirms the hypothesis that variation of the stimulation parameters leads to differences in the PREP results. Overall, our findings revealed that N1P1-amplitudes and evoked pain intensities increase with increasing stimulus duration. However, N1P1-amplitudes remain constant when changing number of stimulation electrodes, while evoked pain intensities decrease when using a higher number of electrodes. The missing dependence between amplitudes and PREP-evoked pain intensities probably indicate that the use of PREP as an objective measure for evoked pain, is limited. However, the present results underlie that PREP could be very well used for small fiber neuropathy studies, especially when a higher number of electrodes can be used to cover a larger area of skin. Most importantly, since our results show that PREP parameters are not comparable when different stimulation protocols are used, a standardization procedure between laboratories should be established.

\section{Declarations}

\section{Acknowledgements}

We are indebted to the subjects who participated in the study for their consent and cooperation.

\section{Author contributions statement}

KB contributed to the study conception, data collection, data analysis and drafted the manuscript, EEK contributed to data analysis and interpretation and revised the manuscript for intellectual content, LE contributed the conception of the study, JV analysed the data, CM contributed the study conception and revised the manuscript for intellectual content, $\mathrm{OH}$ contributed the conception of the study, data analysis and interpretation and revised the manuscript for intellectual contend, MT contributed to the interpretation of data and revised the manuscript for intellectual contend, ÖÖ contributed the study conception, data analysis, drafted parts of the manuscript and revised the manuscript for intellectual content. 


\section{Conflict of interest statement}

The authors declare that the research was conducted in the absence of any commercial or financial relationships. None of the authors have potential conflicts of interest to be disclosed.

\section{Funding}

This work was supported by the Deutsche Forschungsgemeinschaft, SFB 874/A1 and A5, Project No.: 122679504. E. E.-K. holds an endowed professorships funded by the German Social Accident Insurance (DGUV) for the time of 6 years (2020-2026). Ö.S.Ö. received intramural funding from the Ruhr University Bochum, Germany (FoRUM grant nr. K120-18). L.B.E. received intramural funding from the Ruhr University Bochum, Germany (FoRUM grant nr. K K135-19).

\section{References}

1. Perchet, C. et al. Do we activate specifically somatosensory thin fibres with the concentric planar electrode? A scalp and intracranial EEG study., 153 (6), 1244-1252 https://doi.org/10.1016/j.pain.2012.03.004. (2012).

2. Katsarava, Z. et al. A novel method of eliciting pain-related potentials by transcutaneous electrical stimulation. Headache, 46 (10), 1511-1517 https://doi.org/10.1111/j.1526-4610.2006.00446.x. (2006).

3. Obermann, M. et al. Correlation of epidermal nerve fiber density with pain-related evoked potentials in HIV neuropathy., 138 (1), 79-86 https://doi.org/10.1016/j.pain.2007.11.009. (2008).

4. Hansen, N. et al. [Clinical application of pain-related evoked potentials],Schmerz26 (2011)

5. Yoon, M. S. et al. Sensory neuropathy in patients with cryoglobulin negative hepatitis-C infection. $J$. Neurol, 258 (1), 80-88 https://doi.org/10.1007/s00415-010-5686-1. (2011).

6. Ayzenberg, I. et al. Central sensitization of the trigeminal and somatic nociceptive systems in medication overuse headache mainly involves cerebral supraspinal structures., 26 (9), 1106-1114 https://doi.org/10.1111/j.1468-2982.2006.01183.x. (2006).

7. Lauria, G. et al. European Federation of Neurological Societies/Peripheral Nerve Society Guideline on the use of skin biopsy in the diagnosis of small fiber neuropathy. Report of a joint task force of the European Federation of Neurological Societies and the Peripheral Nerve Society, Eur. J. Neurol. 17 (7) (2010) 903-12, e

44-9

. https://doi.org/10.1111/j.1468-1331.2010.03023.x

8. Kakigi, R., Endo, C., Neshige, R., Kuroda, Y. \& Shibasaki, H. Estimation of conduction velocity of A delta fibers in humans. Muscle Nerve, 14 (12), 1193-1196 https://doi.org/10.1002/mus.880141209. (1991). 
9. de Tommaso, M. et al. A comparative study of cortical responses evoked by transcutaneous electrical vs CO(2) laser stimulation. Clin. Neurophysiol, 122 (12), 2482-2487 https://doi.org/10.1016/j.clinph.2011.05.006. (2011).

10. Oh, K. J. et al. Pain-related evoked potential in healthy adults. Ann. Rehabil. Med, 39 (1), 108-115 https://doi.org/10.5535/arm.2015.39.1.108. (2015).

11. Höffken, O., Özgül, Ã. S., Enax-Krumova, E. K., Tegenthoff, M. \& Maier, C. Evoked potentials after painful cutaneous electrical stimulation depict pain relief during a conditioned pain modulation. BMC Neurol, 17, https://doi.org/10.1186/s12883-017-0946-7. (2017).

12. Bogdanov, V. B. et al. Cerebral responses and role of the prefrontal cortex in conditioned pain modulation: An fMRI study in healthy subjects. Behav. Brain Res, 281, 187-198 https://doi.org/10.1016/j.bbr.2014.11.028. (2015).

13. Üçeyler, N. Small fiber pathology-a culprit for many painful disorders? Pain 157 Suppl 1 (2016) S606. https://doi.org/10.1097/j.pain.0000000000000411

14. Hansen, N. et al. Amplitudes of Pain-Related Evoked Potentials Are Useful to Detect Small Fiber Involvement in Painful Mixed Fiber Neuropathies in Addition to Quantitative Sensory Testing - An Electrophysiological Study. Front. Neurol, 6, 244 https://doi.org/10.3389/fneur.2015.00244. (2015).

15. Katsarava, Z. et al. Pain related potentials by electrical stimulation of skin for detection of small-fiber neuropathy in HIV. J. Neurol, 253 (12), 1581-1584 https://doi.org/10.1007/s00415-006-0262-4. (2006).

16. Mueller, D. et al. Electrically evoked nociceptive potentials for early detection of diabetic small-fiber neuropathy. Eur. J. Neurol, 17 (6), 834-841 https://doi.org/10.1111/j.1468-1331.2009.02938.x. (2010).

17. Hagenacker, T. et al. Patient-conducted anodal transcranial direct current stimulation of the motor cortex alleviates pain in trigeminal neuralgia. J. Headache Pain, 15, 78 https://doi.org/10.1186/11292377-15-78. (2014).

18. Üçeyler, N. et al. Impaired small fiber conduction in patients with Fabry disease: A neurophysiological case-control study. BMC Neurol, 13, 47 https://doi.org/10.1186/1471-2377-13-47. (2013).

19. Üçeyler, N. \& Sommer, C. Objective evidence that small-fiber polyneuropathy underlies some illnesses currently labeled as fibromyalgia., 154 (11), 2569 https://doi.org/10.1016/j.pain.2013.06.037. (2013).

20. Üçeyler, N. et al. Small fibre pathology in patients with fibromyalgia syndrome, Brain 136 (Pt 6)(2013) 1857-1867. https://doi.org/10.1093/brain/awt053

21. Siedler, G., Sommer, C. \& Üçeyler, N. Pain-related evoked potentials in patients with large, mixed, and small fiber neuropathy. Clin. Neurophysiol, 131 (3), 635-641 https://doi.org/10.1016/j.clinph.2019.12.006. (2020).

22. Fischer, M., Höffken, O., Özgül, Ã. S. \& Maier, C. Bilaterally prolonged latencies of pain-related evoked potentials in peripheral nerve injuries. Neurosci. Lett, 684, 78-85 https://doi.org/10.1016/j.neulet.2018.07.002. (2018). 
23. Omori, S. et al. Pain-related evoked potentials after intraepidermal electrical stimulation to $A \delta$ and $C$ fibers in patients with neuropathic pain. Neurosci. Res, 121, 43-48 https://doi.org/10.1016/j.neures.2017.03.007. (2017).

24. Özgül, Ã. S. et al. High test-retest-reliability of pain-related evoked potentials (PREP) in healthy subjects. Neurosci. Lett, 647, 110-116 https://doi.org/10.1016/j.neulet.2017.03.037. (2017).

25. Eitner, L. et al. Conditioned pain modulation using painful cutaneous electrical stimulation or simply habituation? Eur. J. Pain, 22 (7), 1281-1290 https://doi.org/10.1002/ejp.1215. (2018).

26. Gierthmühlen, J. et al. Who is healthy? Aspects to consider when including healthy volunteers in QST-based studies-a consensus statement by the EUROPAIN and NEUROPAIN consortia., 156 (11), 2203$2211 \mathrm{https} / / /$ doi.org/10.1097/j.pain.0000000000000227. (2015).

27. Oldfield, R. C. The assessment and analysis of handedness: the Edinburgh Inventory, Neuropsychologia (Vol. 9) (1971) 97-113

28. Eva, L. et al. A Practical Two-Step Quantitative Clinical and Electrophysiological Assessment for the Diagnosis and Staging of Diabetic Neuropathy., (17 (11), 1281-1289 https://doi.org/10.2337/diacare.17.11.1281. (1994).

29. Löwe, B. et al. A 4-item measure of depression and anxiety: Validation and standardization of the Patient Health Questionnaire-4 (PHQ-4) in the general population. J. Affect. Disord, 122 (1-2), 86-95 https://doi.org/10.1016/j.jad.2009.06.019. (2010).

30. Obermann, M. et al. Temporal summation of trigeminal pain in human anterior cingulate cortex., 46 (1), 193-200 https://doi.org/10.1016/j.neuroimage.2009.01.038. (2009).

31. Truini, A. et al. Laser-evoked potentials: Normative values. Clin. Neurophysiol, 116 (4), 821-826 https://doi.org/10.1016/j.clinph.2004.10.004. (2005).

32. lannetti, G. D. et al. Adelta nociceptor response to laser stimuli: Selective effect of stimulus duration on skin temperature, brain potentials and pain perception. Clin. Neurophysiol, 115 (11), 2629-2637 https://doi.org/10.1016/j.clinph.2004.05.023. (2004).

33. Obermann, M. et al. Impaired trigeminal nociceptive processing in patients with trigeminal neuralgia. Neurology, 69 (9), 835-841 https://doi.org/10.1212/01.wnl.0000269670.30045.6b. (2007).

34. Banerjee, S. \& Muthuswamy, B. Introduction to Nonlinear Circuits and Networks 1st edn (Springer International Publishing, Springer, Cham, 2018).

\section{Figures}




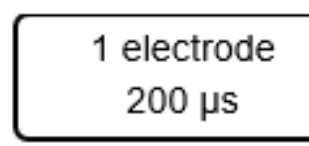

Group A
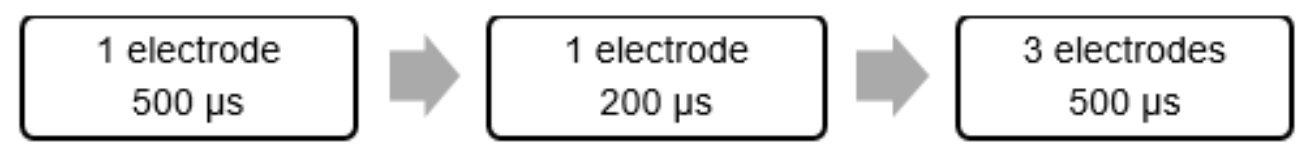

\section{Group B}
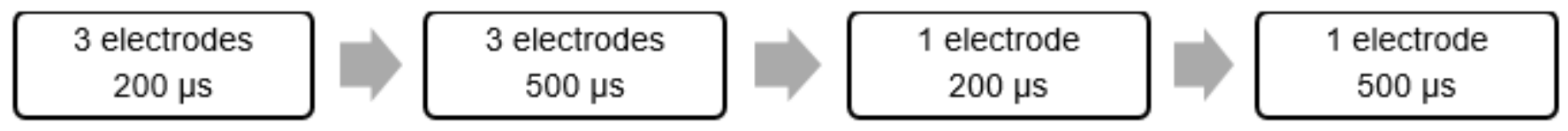

\section{Group C}
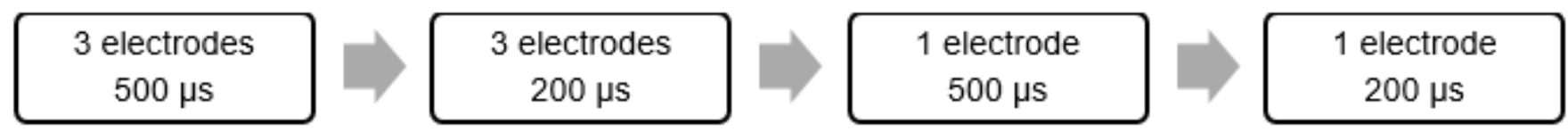

\section{Group D}

\section{Figure 1}

Randomisation of stimulus combinations into groups A-D 


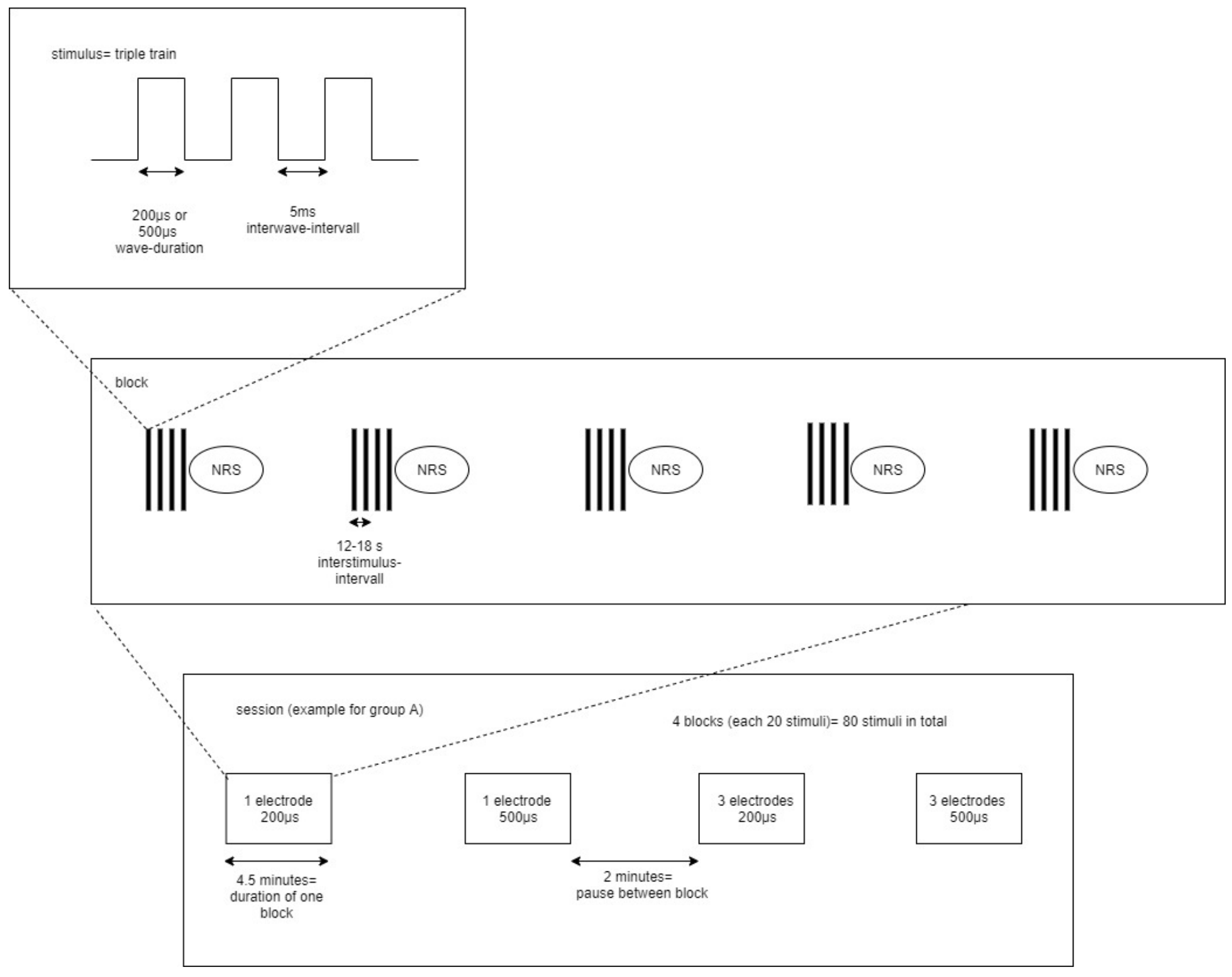

Figure 2

Stimulation paradigm: 4 blocks of 20 stimuli each (duration $4.5 \mathrm{~min}$ ) at current intensities of twofold of the pain threshold, separated by 2 min pause, were delivered. After every forth stimulus participants had to rate the pain perceived on the NRS (0-100). Each of the 20 stimuli consisted of a triple train, where each pulse lasted either $200 \mu \mathrm{s}$ or $500 \mu \mathrm{s}$, followed by an interwave-interval of 5 ms with a randomised interstimulus interval of 12-18s 


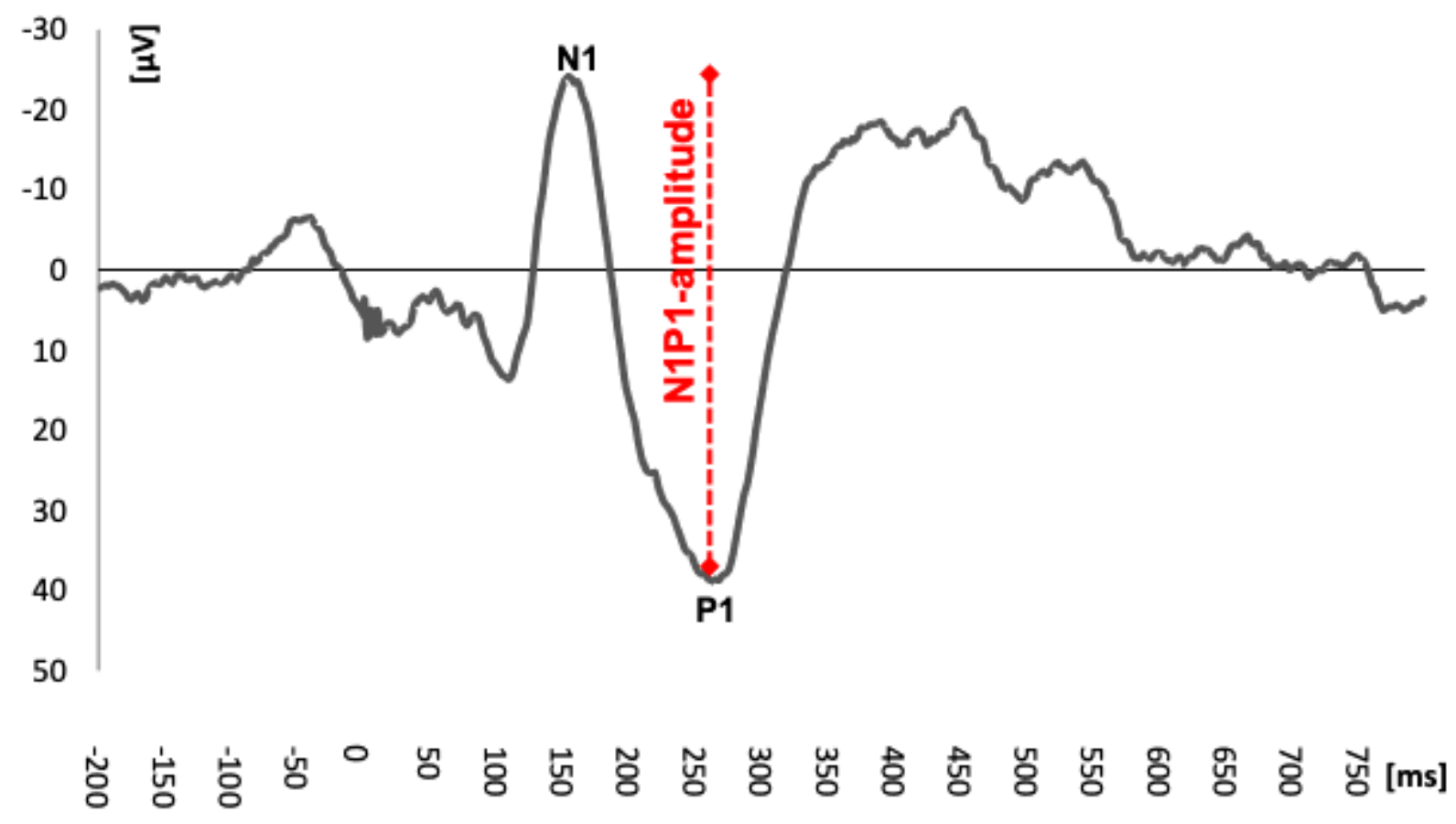

Figure 3

PREP recording with positive (P1) and negative (N1) peaks 